\title{
A Computer Model of Reflection Induced by Early Afterdepolarizations in Ventricular Tissue
}

\author{
E Ramirez ${ }^{1,2}, \mathrm{~J} \mathrm{Saiz}^{2}, \mathrm{M}_{\text {Monserrat }}{ }^{2}, \mathrm{JM}$ Ferrero $(\mathrm{Jr})^{2}$, \\ $\mathrm{J} \mathrm{Gomis-Tena}^{2}$, V Hernandez ${ }^{3}$ \\ ${ }^{1}$ Departamento de Ingenierías, Instituto Tecnológico de Cuautla, Morelos, México \\ ${ }^{2}$ Center for Research and Innovation in Bioengineering, Universidad Politécnica de Valencia, Spain \\ ${ }^{3}$ Grupo de Redes y Computación de Altas Prestaciones, Universidad Politécnica de Valencia, Spain
}

\begin{abstract}
A computer model has been developed in which reflection was observed. Reflection is a type of reentry in a one-dimensional structure of cardiac cells. The model consists of two segments of ventricular tissue partially coupled by a coupling resistance $(R)$ and it was used to study conditions that provoke the development of EADs, and the generation of reflection and ectopic activity. In the present study, EADs were induced in one segment by enhancing $I_{C a L}$ current and decreasing $I_{K r}$ and $I_{K s}$ currents. The other segment was maintained under normal physiological conditions. The results suggest that ectopic activity in a zone of myocardium could appear due to the reflection induced by EADs generated in neighboring tissue zones. The coupling between areas and the blocked factor of the two components of the delayed rectifier $K^{+}$current $\left(I_{K r}\right.$ and $\left.I_{K s}\right)$, have a great influence. The results suggest that a partial coupling between segments and severe conditions in the abnormal segment increase the likelihood of both the ectopic activity and the triggered activity.
\end{abstract}

\section{Introduction}

Reflection is a phenomenon that has been observed in ventricular tissue in which an impulse reaches an abnormal area and induces another impulse that returns in the direction from which the first impulse came ("reflected" impulse) [1]. Reflection, also named "reflected" reentry, is a special type of reentrant circuit in a one-dimensional structure. The structure is normally divided in two segments (proximal and distal) joined by a zone with depressed excitability. When propagation of impulses in both directions is over the same fibers, reflection is called true reflection [2]. Reflection has been demonstrated in computer models, and in experimental models of ventricular and Purkinje fibers [1-4]. In experiments performed by Rozanski, Jalife and Moe, in isolated Purkinje fibers and in non homogeneous ventricular tissue, the authors used a three-chambered tissue bath in which a zone of conduction block was created in the central chamber, representing an inexcitable gap or an electrical insulation. The mechanism they proposed for reflection was that if the delay across the gap is long enough to permit recovery of refractoriness at proximal segment, electrotonic potential of the distal segment can reexcite the proximal fiber [4]. Wit and coworkers observed reflection in Purkinje fibers with a central segment with depressed excitability, and they suggested that reflection was produced by microreentry [5].

Early afterdepolarizations (EADs) are subthreshold depolarizations that occur in phases 2 and 3 of the action potential (AP), causing interruption or delay in repolarization [6-8]. January et al. suggested that induction of EADs require the prolongation of the action potential plateu and the recovery of the L-type calcium current $\left(\mathrm{I}_{\mathrm{CaL}}\right)$ [9]. Some experimental studies have shown that EADs generated in Purkinje fibers propagate to ventricular cells, inducing ectopic activity [8]. On the other hand, in vitro studies suggested that EADs may be responsible for some arrhythmias by prolonging the action potential, generating injury currents and inducing triggered activity [10]. Patients with long QT syndrome (LQTS) have greater probability of developing torsades de pointes (TdP), possibly triggered by EADs arising from Purkinje cells or ventricular tissue [11].

The main goal of this work has been to study conditions in which EADs developed in an area of ventricular myocardium induce reflection to neighboring zones.

\section{Methods}

We performed a series of simulations with a onedimensional computer model formed by two segments of ventricular cells, normal segment (NS) and abnormal segment (AS), 100 cells each one. The segments were partially coupled by a coupling resistance (R). R represents a tissue with depressed excitability and was 
varied in a range from 5 to $40 \Omega \mathrm{cm}^{2}$. Stimuli are applied to cell \#0 (NS). NS was kept under normal physiological conditions while AS was set under conditions that favor the induction of EADs (figure 1). The generation of EADs is favored by conditions that lengthen action potential duration (APD), such as conditions that increase an inward current or decrease an outward current [8]. We blocked the components of the delayed rectifier $\mathrm{K}^{+}$ current, $\mathrm{I}_{\mathrm{Kr}}$ and $\mathrm{I}_{\mathrm{Ks}}$, in the range from $0 \%$ to $100 \%$ while the L-type $\mathrm{Ca}^{2+}$ current $\left(\mathrm{I}_{\mathrm{caL}}\right)$ was incremented to $100 \%$ in order to probe different EAD conditions.

Simulations utilized the Luo-Rudy (LRd00) model of the mammalian cardiac ventricular cells to calculate action potential (AP) [12,13]. A finite-difference technique in space and time using the implicit method of Crank and Nicholson was used to solve the differential equation that describes AP propagation. For steady state conditions, 10 stimuli were applied at a basic cycle length (BCL) of $1000 \mathrm{~ms}$. A final stimulus (11-th stimulus) was applied to obtain AP characteristics at cells \#49, \#100 and \#149 of the fiber. A value of $5 \Omega \mathrm{cm}^{2}$ was used for the intercellular resistance between ventricular cells. The amplitude of impulses was 1.2 times diastolic threshold, with a duration of $1 \mathrm{~ms}$.

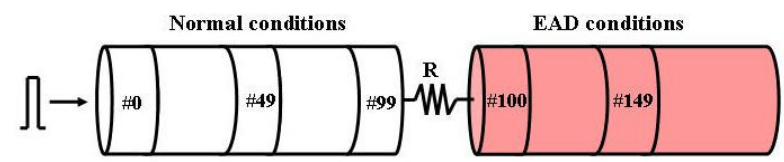

Figure 1. The model of the fiber is compound of two segments (normal and abnormal segments), 100 cells each one. The segments are joined by a coupling resistance $\mathrm{R}$.

\section{Results}

Lengthening of the action potential duration (APD) and different kinds of EADs appear in the cells of the abnormal segment that provoke three different situations in the cells of the normal segment: multiple reflected ectopic beats (Reflected Triggered Activity, RTA), a single reflected beat (SRB) and none reflected activity.

When the segments were coupled with a coupling resistance of $5 \Omega \mathrm{cm}^{2}$, and $\mathrm{I}_{\mathrm{Ks}}$ was blocked $80 \%, \mathrm{I}_{\mathrm{Kr}} 20 \%$ and $\mathrm{I}_{\mathrm{CaL}}$ was incremented $100 \%$, we observed two ectopic beats (RTA) in the normal segment induced by EADs formed in abnormal segment (see cell \#49, figure 2A). If we increase $\mathrm{R}$ to $10 \Omega \mathrm{cm}^{2}$, the ectopic activity also increases, now we observed three ectopic beats (RTA) in the normal segment (see cell \#49, figure 2B). A further increase in the coupling resistance $\mathrm{R}\left(20 \Omega \mathrm{cm}^{2}\right)$ reduced the ectopic activity (figure $2 \mathrm{C}$ ). In this case, we obtained only one ectopic beat (SRB) that was induced by EADs.
If we continue increasing $\mathrm{R}$, the ectopic activity disappears (not shown in figure 2). The ectopic activity was influenced by the coupling resistance and the conditions of abnormal segment. An intermediate coupling and severe conditions in $\mathrm{I}_{\mathrm{Ks}}$ and $\mathrm{I}_{\mathrm{Kr}}$ blocking factors influenced the probability of reflection. Also, with severe conditions that favored EAD induction, ectopic activity and reflection occurred for coupling values near normal ones $\left(5 \Omega \mathrm{cm}^{2}\right)$.

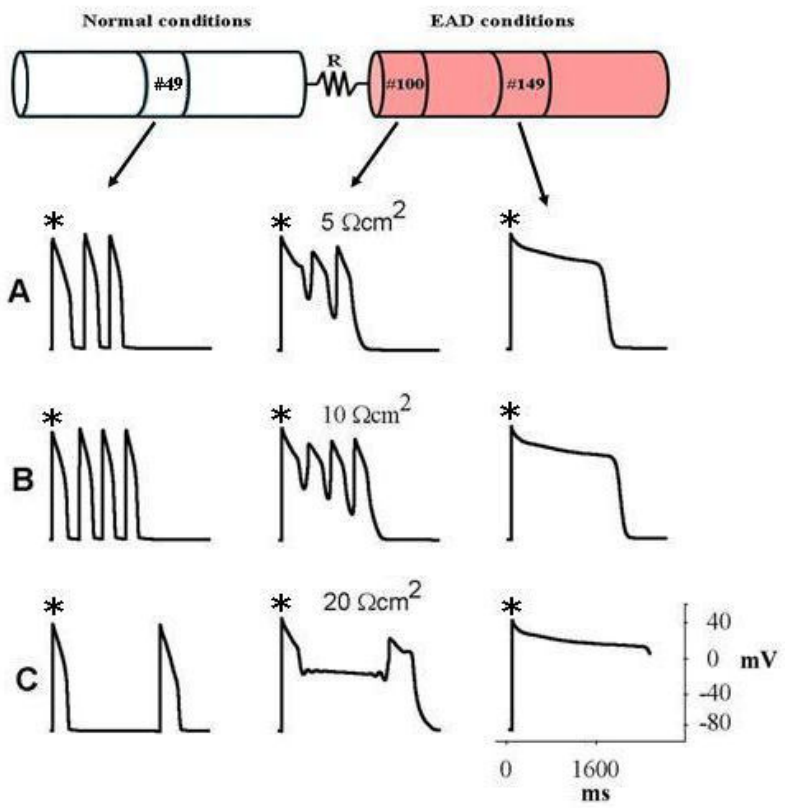

Figure 2. Influence of coupling resistance on Reflection. Action potentials marked with an asterisk (*) were obtained when the last (11-th) stimulus was applied to the fiber. $\mathrm{R}$ was varied in a range from 5 to $40 \Omega \mathrm{cm}^{2}$. $\mathrm{I}_{\mathrm{Kr}}$ was blocked $20 \%$, $\mathrm{I}_{\mathrm{Ks}}$ was blocked $80 \%$ and $\mathrm{I}_{\mathrm{CaL}}$ was enhanced $100 \%$.

When more severe conditions were maintained in the abnormal segment, the ectopic activity and reflection were greater. In figure 3 , we used a constant coupling of $10 \Omega \mathrm{cm}^{2}, \mathrm{I}_{\mathrm{Ks}}$ was blocked by $60 \%, \mathrm{I}_{\mathrm{CaL}}$ enhanced by $100 \%$ and $\mathrm{I}_{\mathrm{Kr}}$ blocked in a range from 0 to $100 \%$. In the case of $\mathrm{I}_{\mathrm{Kr}}$ severe blockade, multiple EADs were induced in the abnormal segment that provoked several ectopic action potentials in the normal segment (see figure $3 \mathrm{~A}$ ). Action potential duration (APD) of cell \#149 was near 2250 ms. Reduction in $\mathrm{I}_{\mathrm{Kr}}$ blockade reduced $\mathrm{EAD}$ induction and ectopic activity and the frequency of ectopic beats in cell \#49 was lower (not shown in figure 3). When $\mathrm{I}_{\mathrm{Kr}}$ was blocked $20 \%$, only one EAD was observed in abnormal fiber, and an ectopic beat was induced and reflected. When $\mathrm{I}_{\mathrm{Kr}}$ current was not blocked 
APD was reduced and an EAD was induced without a second depolarization (figure 3C). Ectopic activity and reflection were not observed for these conditions, when we used a greater $\mathrm{I}_{\mathrm{Ks}}$ blockade (greater than $90 \%$ ) and a coupling resistance near $10 \Omega \mathrm{cm}^{2}$, we obtained reflection for any value of $\mathrm{I}_{\mathrm{Kr}}$ blockade (not shown in figure 3 ).

In figure 4 action potentials obtained when $\mathrm{I}_{\mathrm{Kr}}$ current and the coupling resistance $(\mathrm{R})$ were maintained constant are shown. $\mathrm{I}_{\mathrm{Kr}}$ was blocked $60 \%, \mathrm{I}_{\mathrm{CaL}}$ was incremented $100 \%$ and $\mathrm{R}$ was fixed to $10 \Omega \mathrm{cm}^{2}$. In figure $4 \mathrm{~A}, \mathrm{I}_{\mathrm{Ks}}$ was blocked $80 \%$. It can be observed a very prolonged APD and EADs that provoked multiple depolarization peaks that induced reflection (reflected triggered activity, RTA). Reduction of $\mathrm{I}_{\mathrm{Ks}}$ blockade to $20 \%$, shortened the APD approximately to $640 \mathrm{~ms}$, but ectopic beats were not induced in the normal segment (see figure 4B). If we do not block the $\mathrm{I}_{\mathrm{Ks}}$ current, the APD is lengthened, but not enough to provoke ectopic activity and reflection in the normal segment (none reflected activity), as it is shown in figure $4 \mathrm{C}$.

As it is observed in cell \#49 of figures $3 \mathrm{~A}$ and $4 \mathrm{~A}$, reflected triggered activity (RTA) was obtained when $\mathrm{I}_{\mathrm{Ks}}$ and $\mathrm{I}_{\mathrm{Kr}}$ were blocked $80 \%$. In the case of $\mathrm{I}_{\mathrm{Ks}}$ and $\mathrm{I}_{\mathrm{Kr}}$ blockade of $20 \%$, only $\mathrm{I}_{\mathrm{Kr}}$ blockade generate ectopic activity in the normal segment (cell \#49, figures $3 \mathrm{~B}$ and 4B).
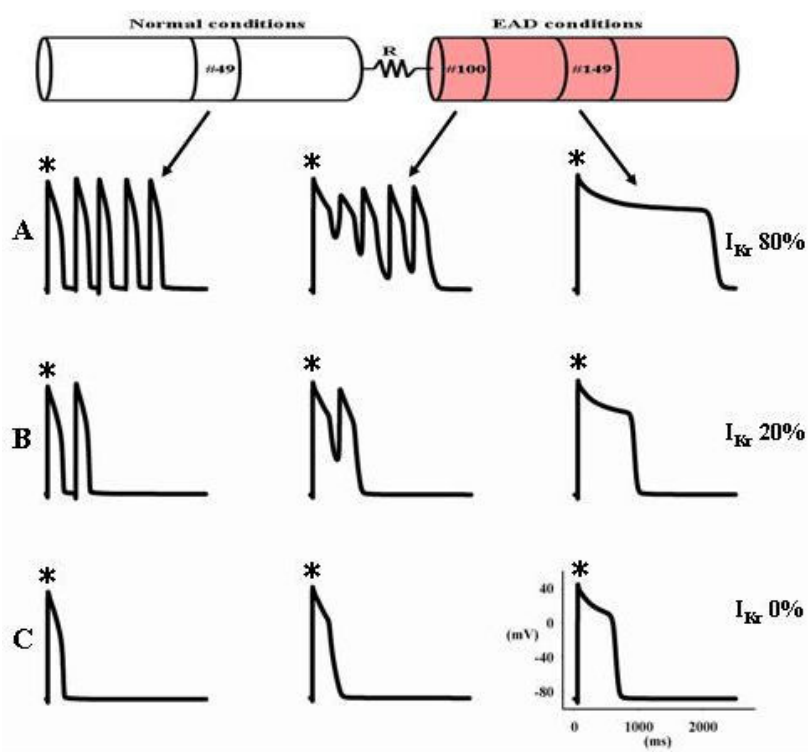

Figure 3. Influence of $\mathrm{I}_{\mathrm{Kr}}$ blockade on reflection. Action potentials marked with an asterisk (*) were obtained when the last (11-th) stimulus was applied to the fiber. $\mathrm{I}_{\mathrm{Ks}}$ was blocked $60 \%, \mathrm{I}_{\mathrm{CaL}}$ was incremented $100 \%$ and a coupling resistance $(\mathrm{R})$ of $10 \Omega \mathrm{cm}^{2}$ was utilized.

\section{Discussion and conclusions}

The results have shown that coupling between tissue regions has a fundamental role in reflection. Saiz et al. found that for low values of R, strong EAD conditions are necessary for reflection [1]. Our results are in agreement with this conclusion. On the other hand, the greater the $\mathrm{I}_{\mathrm{Ks}}$ and $\mathrm{I}_{\mathrm{Kr}}$ blockade the higher the probability of reflected triggered activity (RTA) and single reflected beat (SRB). Cheng and Kodama suggest that a lengthening of action potential duration (APD) would favor $\mathrm{I}_{\mathrm{Ks}}$ activation to prevent excessive APD and generation of EADs. In most mammalian, $\mathrm{I}_{\mathrm{Kr}}$ current has a main role in normal ventricular repolarization [14]. The results obtained in our study indicate that both currents have a great influence on reflection, but perhaps, a greater influence of the $\mathrm{I}_{\mathrm{Ks}}$ current could exist (see figures $3 \mathrm{~B}$ and $4 \mathrm{~B}$ ).
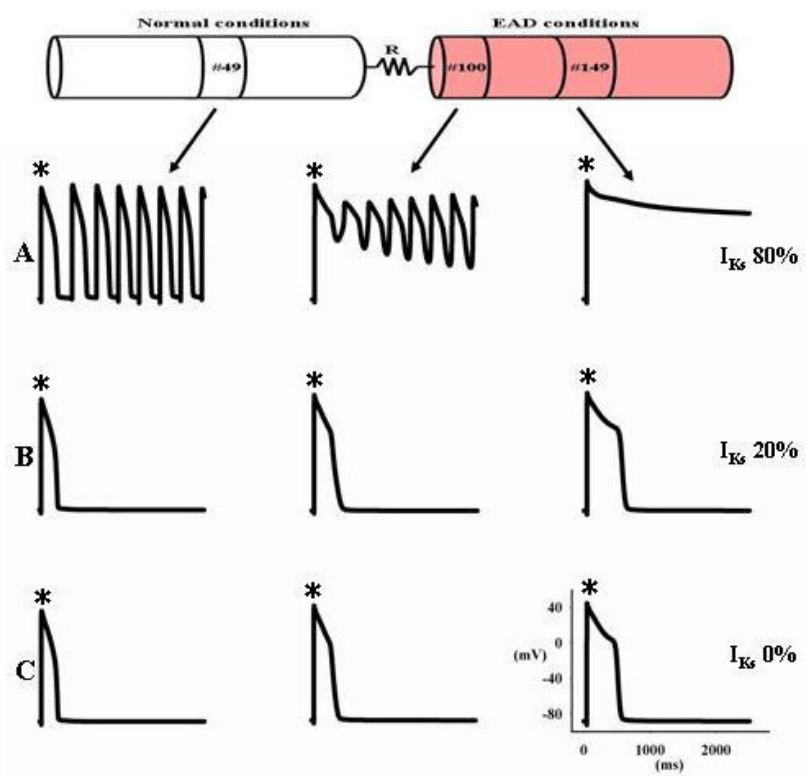

Figure 4. Influence of $\mathrm{I}_{\mathrm{Ks}}$ blockade on reflection. Action potentials marked with an asterisk (*) were obtained when the last (11-th) stimulus was applied to the fiber. $\mathrm{I}_{\mathrm{Kr}}$ was blocked $60 \%, \mathrm{I}_{\mathrm{CaL}}$ was incremented $100 \%$ and a coupling resistance of $10 \Omega \mathrm{cm}^{2}$ was used.

Cabo and Barr found a narrower coupling range where reflection occurred than ours in Purkinje fibers [2]. They obtained a delay between distal and proximal segments for reflection of approximately $300 \mathrm{~ms}$. Whereas the two segments of our model had a delay that was much smaller than this value.

In conclusion, the study shows that reflection in a zone of myocardium could appear due to EADs induced in neighboring zones. The coupling between areas and the 
blockade of $\mathrm{I}_{\mathrm{Ks}}$ and $\mathrm{I}_{\mathrm{Kr}}$ currents have a great influence. A partial coupling between areas increases the likelihood of both ectopic and triggered activity.

\section{Acknowledgements}

This work was partially supported by the Plan Nacional de Investigación Científica, Desarrollo e Innovación Tecnológica del Ministerio de Educación y Ciencia of Spain (TIC 2001-2686, TIC 2004-03602) and by the Programa Incentivo a la Investigación de la Univ. Politécnica de Valencia (CAMAV 20020418).

\section{References}

[1] Saiz J, Monserrat M, Gomis J, Torres V, Ferrero JM (Jr), Ferrero JM, Thakor NV. Reflection and ectopic activity induced by early afterdepolarizations. A computer modeling study. 20th Annual International Conference IEEE/EMBS 1998, Hong Kong:IEEE, 1998:3090-3093.

[2] Cabo C, Barr, RC. Reflection after delayed excitation in a computer model of a single fiber. Circulation Research 1992; 71:260-270.

[3] Rozanski GJ, Jalifé J, Moe GK. Reflected reentry in nonhomogeneous ventricular muscle as a mechanism of cardiac arrhythmias. Circulation 1984; 69:163-173.

[4] Jalife J, Moe GK. Excitation, conduction, and reflection of impulses in isolated bovine and canine cardiac purkinje fibers. Circulation Research 1981; 49:233-247.

[5] Wit AL, Cranefield PF, Hoffman BF. Slow conduction and reentry in the ventricular conducting system: II. Single and sustained circus movement in networks of canine and bobine Purkinje fibers. Circulation Research 1972; 30:1122.

[6] Zeng J, Rudy Y. Early afterdepolarizations in cardiac myocytes: Mechanism and rate dependence. Biophysical Journal 1995; 68:949-964.

[7] Wagner MB, Gibb WJ, Lesh MD. A model study of propagation of early afterdepolizations. IEEE Transactions on Biomedical Engineering 1995; 42:991-997.

[8] Monserrat M, Saiz J, Ferrero JM (Jr), Ferrero JM, Thakor
NV. Ectopic activity in ventricular cells induced by early afterdepolarizations developed in Purkinje cells. Annals of Biomedical Engineering 2000; 28:1343-1351.

[9] January CT, Riddle JM. Early afterdepolarizations: mechanism of induction and block. A role for L-type $\mathrm{Ca}^{2+}$ current. Circulation Research 1989; 64:977-990.

[10] Saiz J, Ferrero JM (Jr), Monzerrat M, Ferrero JM, Thakor $\mathrm{NV}$. Influence of electrical coupling on early afterdepolarizations in ventricular myocytes. IEEE Transactions on Biomedical Engineering 1999; 46:138147.

[11] Ueda N, Zipes DP, Wu J. Prior ischemia enhances arrhythmogenicity in isolated canine ventricular wedge model of long QT 3. Cardiovascular Research 2004; 63:6976.

[12] Luo CH, Rudy Y. A dynamic model of the cardiac ventricular action potential: I. Simulations of ionic currents and concentration changes. Circulation Research 1994; 74:1071-1096.

[13] Faber GM, Rudy Y. Action potential and contractibility changes in $[\mathrm{Na}(+)](\mathrm{i})$ overloaded cardiac myocytes: a simulation study. Biophysics Journal 2000; 78:2392-2404.

[14] Cheng JH, Kodama I. Two components of delayed rectifier $\mathrm{K}+$ current in heart: molecular basis, functional diversity, and contribution to repolarization. Acta Pharmacologica Sinica 2004; 25:137-145.

[1] Janse MJ, D'alnoncourt CN. Reflections on Reentry and focal activity. The American Journal of Cardiology 1987; 60:21F-26Fferences here. With the reference style, numbering is supplied automatically.

Address for correspondence

Esteban Benito Ramírez Islas

Center for Research and Innovation in Bioengineering.

Universidad Politécnica de Valencia.

Camino de Vera s/n, 46022 Valencia, Spain.

esrais@doctor.upv.es 